\title{
Study on Preparation and Dyeing Wastewater Adsorption Properties of
}

\section{Carbonized Cotton}

\author{
Ju WEI ${ }^{1, a}$, Meng Xue YAN ${ }^{1, b}$, Song Jia $L^{1, c}$ and Dan HUANG ${ }^{1, d}$ \\ ${ }^{1}$ School of Textile and Material Engineering, Dalian Polytechnic University, Dalian 116034, China

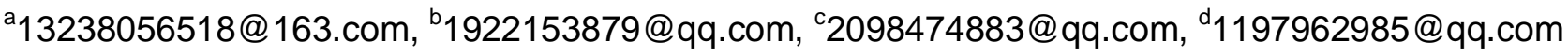

Keywords: waste textiles, activated carbon, dyeing wastewater, cotton fabric, adsorbent.

Abstract. Carbonized waste cotton were prepared by activate solution carbonization method. The effects of moisture content, carbonization time and carbonization temperature on the yield of carbonization were investigated. At the same time, the adsorption property of direct dye of the carbonized cotton products was studied. Results show that carbonization yield increased first and then decreased with the increasing of the moisture content using pure water as the activate solution at the same other conditions; Carbonization yield decreased as the extension of carbonization temperature and carbonization time. Carbonization yield and adsorption of direct dye increased when using phosphoric acid solution as the activate solution. Adsorption of direct dye increased with the increasing of phosphoric acid concentration.

\section{Introduction}

Pollution caused by textile wastewater is a common problem faced by many industrial countries ${ }^{[1]}$. Textile wastewater contains various types of dye which can be toxic to some microorganisms and may cause direct destruction or inhibition of their metabolism ${ }^{[2]}$. Industry already employs adsorbents to strip contaminants from wastewater before it is discharged ${ }^{[3]}$. It is attractive to find cheaper and more effective adsorbents ${ }^{[4]}$. About 400 thousand tons of waste cotton and cotton fabrics are produced in China every year. Those waste cotton textiles were usually discarded directly thus will cause environmental pollution. In this paper, we studied the method of preparing activated carbon from waste cotton fabrics, thus can not only reduced the cost but also diminish the pollution from waste textile.

\section{Experiment}

Equipment. SX2 4-13 high temperature furnace, tianjin Youli technology co., LTD; HCT - 2 microcomputer differential thermal balance constant scientific instrument factory (Beijing); V 5000 type spectrophotometer (Shanghai yuanxi instrument co., LTD.)

Materials. waste cotton fabrics, phosphoric acid, ammonium polyphosphate (APP), distilled water, direct peach dye.

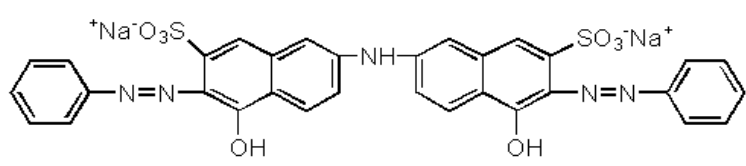

Molecular structure of direct pink 
The preparation of carbonized cotton. The dried cotton fabrics were impregnated in aqueous activating solution of certain concentration with a certain period of time, and then the fabric was baked in the oven until it reached certain moisture content $M$. The above prepared cotton fabrics were carbonized at the set temperature in muffle furnace with a certain time. The carbonization yield $Y$ was calculated from the mass of the carbonized product and that of the dry cotton.

$$
\begin{aligned}
& M=\frac{\left(\mathrm{G}_{I}-\mathrm{G}_{0}\right) \times 100}{\mathrm{G}_{1}}(\%) \\
& Y=\frac{\mathrm{G}_{2} \times 100}{\mathrm{G}_{0}}(\%) \\
& M \quad \text {------- } \text { moisture content } \\
& Y \quad----- \text { carbonization yield } \\
& \mathrm{G}_{0} \text {------- } \quad \text { mass of the dry cotton fabric } \\
& \mathrm{G}_{1} \text {-------- } \quad \text { mass of the wet cotton fabric } \\
& \mathrm{G}_{2} \text {-------- } \quad \text { mass of the carbonized cotton }
\end{aligned}
$$

Adsorption experiments of carbonized cotton on dyeing wastewater. 0.2 grams of carbonized cotton was put into $40 \mathrm{ml}$ direct peach dye solution for 8 hours. The dye adsorption amount was calculated by formula (3) show as follows.

$$
\mathrm{q}=\frac{\left(\mathrm{C}_{0}-\mathrm{C}\right) \cdot \mathrm{v}}{\mathrm{m}}(\%)
$$

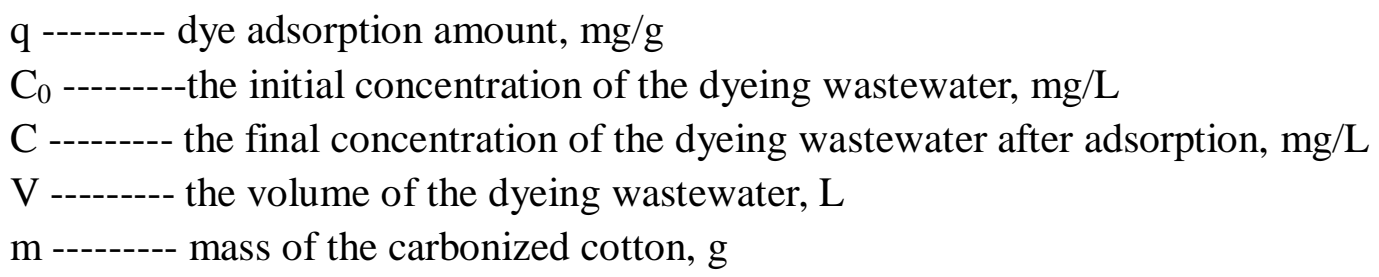

\section{Results and discussion}

Effects of the moisture content on carbonization yield. Cotton fabric will combust under high temperature and oxygen conditions, moisture and closed system can inhibit combustion. The cotton fabrics of different moisture content were carbonized under high temperature. Relationship between moisture content and carbonization yield is shown in Fig. 1.

Fig. 1 shows that with the increasing of moisture content, carbonization yield first increases then decreases. The highest carbonization yield reached at $60 \%$ moisture content.

Effects of carbonization temperature on carbonization yield. The carbonization effect depends on temperature largely, in order to study the effects of carbonization temperature on yield, the cotton fabrics were carbonized under different temperature. Relationship between carbonization temperature and yield is shown in Fig. 2

Fig. 2 shows that with the increasing of carbonization temperature, carbonization yield decreased. This can be explained that the higher the temperature, the more the thermal decomposition. But cotton fabric cannot be carbonized completely with low temperature, under experimental condition of this paper, the cotton fabric treated at $400^{\circ} \mathrm{Cfor} 20 \mathrm{~min}$ can be carbonized completely. 


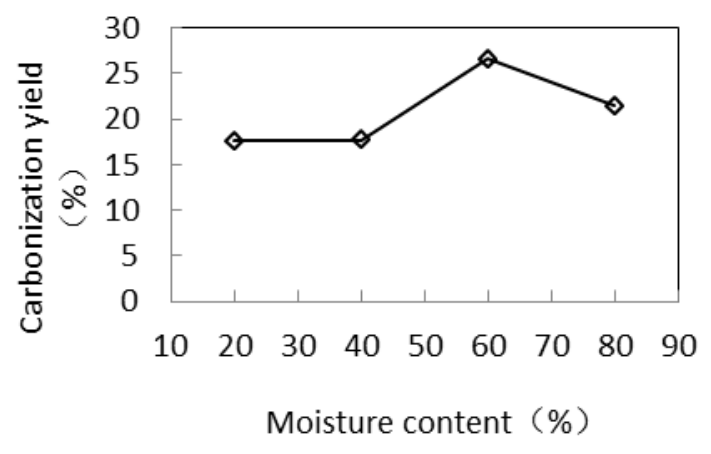

Fig. 1 Relationship between moisture content and carbonization yield

$\left(400^{\circ} \mathrm{C}, 20 \mathrm{~min}\right)$

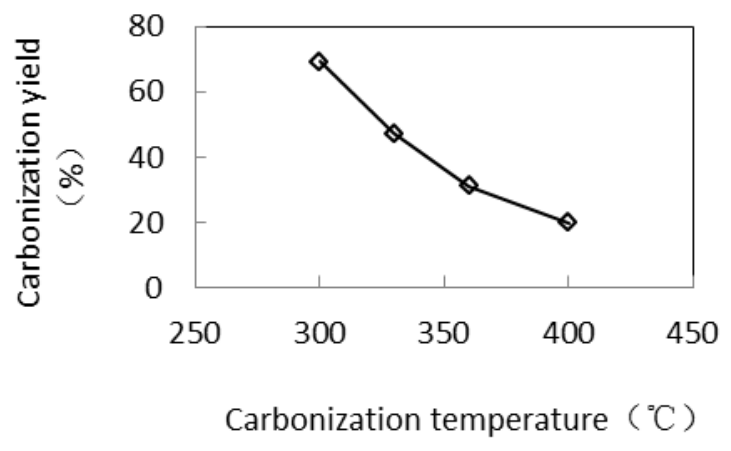

Fig. 2 Relationship between carbonization yield and carbonization temperature

(60\% moisture content, $20 \mathrm{~min}$ )

Effects of carbonization time on carbonization yield. Cotton fabric will take a series of chemical reactions and turns to carbon materials finally under high temperature condition. The chemical reactions will take a period of time. In order to study effects of carbonization time on yield, cotton fabrics were carbonized under different time span, Relationship between carbonization time and yield is shown in Fig. 3.

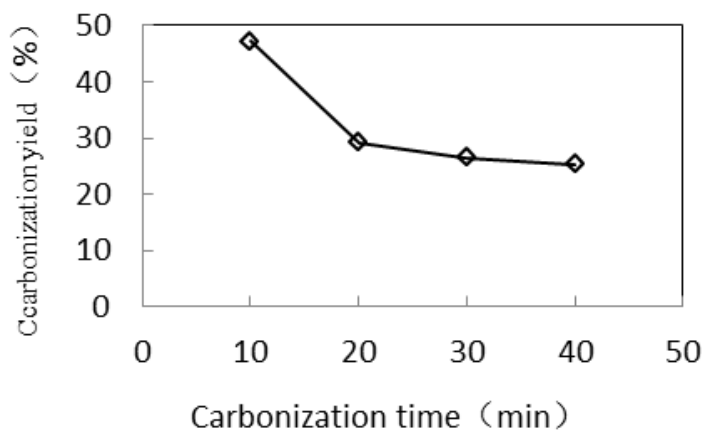

Fig. 3 Relationship between carbonization yield and carbonization time

(400ํㄷ, $60 \%$ moisture content)

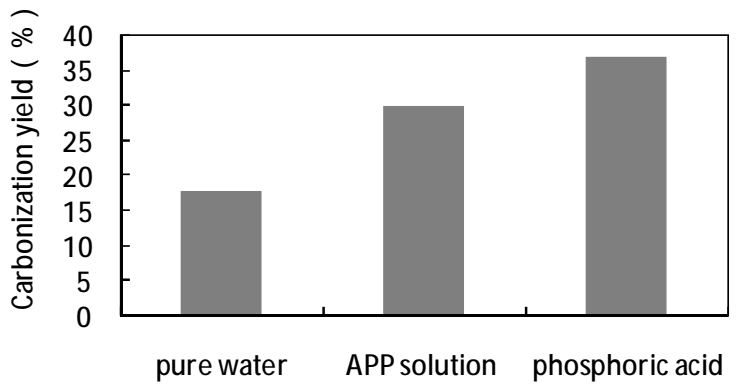

Fig. 4 Effects of activate agent type on carbonization yield

(20\% concentration of activate solution , $60 \%$ moisture content, $\left.400^{\circ} \mathrm{C}, 20 \mathrm{~min}\right)$

Fig. 3 shows that with the extension of carbonization time, the carbonization yield reduced gradually. This can be explained that cotton fabric will take a certain degree of cyclization oxidation under high temperature, elements such as hydrogen and oxygen will turn to small molecule gas and carbon will deposited gradually. The longer the time, the less the other elements in the fiber, and the lower the carbonization yield. But the carbonization cannot finish completely when time is too short. Under experimental condition of this paper, the cotton fabric treated for 20 min can be carbonized completely.

Effects of activate solution type on carbonization yield. Carbonization yield of cotton fabrics activated by pure water is low. In order to improve the carbonization yield, a certain amount 
of chemicals were put into the aqueous activate solution. Both phosphoric acid and ammonium polyphosphate have some flame retardant effect on cotton fabrics, can promote cotton fabric generate carbon under high temperature. Carbonization yields of cotton fabrics activated by three different type of activation solution were shown in Fig. 4.

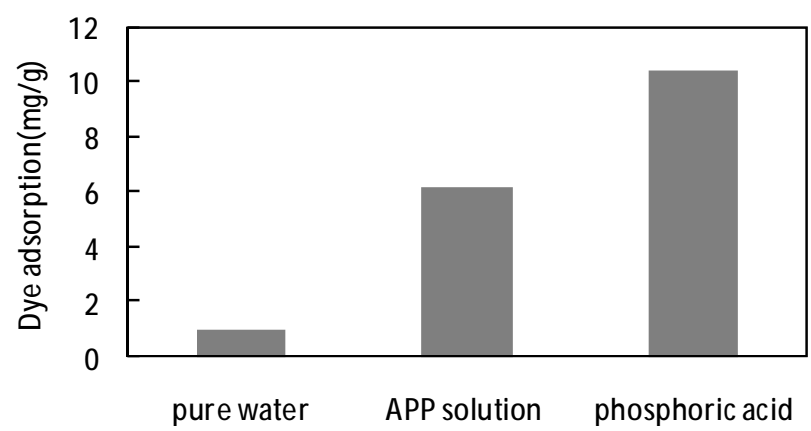

Fig. 5 Effects of activate agent type on dye adsorption

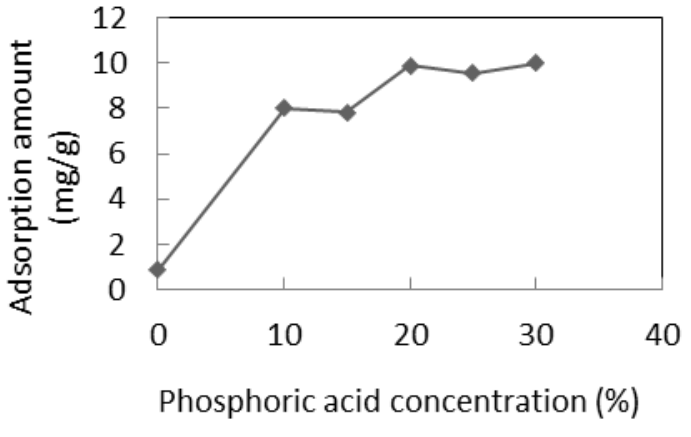

Fig. 6 Effects of activator phosphate content on adsorption property of the carbonized cotton

Effects of activate solution type on adsorption amount. Direct pink dye adsorption experiment was carried on under the same conditions to investigate effects of activator type. Direct pink dye adsorption amount was shown in Fig. 5.

Fig. 5 shows that direct pink adsorption of carbonized cotton activated by phosphoric acid aqueous solution is the highest; adsorption of carbonized cotton activated by pure water is the lowest. The results indicated that adding a certain amount of activator is beneficial to further improve the adsorption performance of carbonized cotton.

Effects of activator phosphate content on adsorption property of the carbonized cotton. Fig. 6 shows that with the increasing of phosphate concentration, adsorption quantity of direct pink dye of the carbonized cotton increased gradually and tend to saturation.

\section{Conclusions}

Carbonized waste cotton were prepared by activate solution carbonization method. Results show that carbonization yield increased first and then decreased with the increasing of the moisture content using pure water as the activate solution at the same other conditions; Carbonization yield decreased as the extension of carbonization temperature and carbonization time. Carbonization yield and adsorption of direct dye increased when using phosphoric acid solution as the activate solution. Adsorption of direct dye increased with the increasing of phosphoric acid concentration.

\section{Acknowledgements}

This work was financially supported by the Liaoning provincial innovative training program of college students and innovative program of Dalian Polytechnic University.

\section{Literature References}

[1] I. Oliveira, D. Blöhse, H.G. Ramke: Bioresource technology Vol. 142 (2013), p. 138

[2] Rashmi Sanghi, Preeti Verma: Color. Technol., Vol.129 (2013), p. 85

[3] J.V. Romero, J.W.H, Smith, C.L. White, S. Trussler, L.M. Croll, J.R. Dahn: J. Hazard. Mater 
Vol.183 (2010), p. 677

[4] S. Maiti, S. Purakayastha, B. Ghosh: Chem. Eng. Comm., Vol.195 (2008), p.386 